\title{
A THEOREM ON SURFACE AREA
}

\author{
BY \\ R. G. HELSEL
}

\section{INTRODUCTION}

0.1. In this paper we shall be concerned with Frécheț surfaces of the type of the 2-cell $[9$, chap. I $]\left({ }^{1}\right)$. Such a surface admits of a representation of the form

$$
T: \quad x=x(u, v), \quad y=y(u, v), \quad z=z(u, v), \quad(u, v) \in D,
$$

where $D$ is the unit disc $u^{2}+v^{2} \leqq 1$ and the transformation $T$ is continuous on $D$. The Lebesgue area of the surface determined by $T$ will be denoted by $A(T)$. Of course this area is independent of the choice of representation for the surface $[10$, p. 6$]$.

Let a second Fréchet surface be given by a representation

$$
T_{*}: \quad x=x_{*}\left(u_{*}, v_{*}\right), \quad y=y_{*}\left(u_{*}, v_{*}\right), \quad z=z_{*}\left(u_{*}, v_{*}\right), \quad\left(u_{*}, v_{*}\right) \in D_{*},
$$

where $D_{*}$ is the unit disc $u_{*}^{2}+v_{*}^{2} \leqq 1$ and $T_{*}$ is continuous on $D_{*}$. If the Lebesgue area of this second surface is denoted by $A\left(T_{*}\right)$, then the question arises:

How should the two representations $T$ and $T_{*}$ be related in order for $A(T)$ to equal $A\left(T_{*}\right)$ ?

0.2 . It is well known that $A(T)=A\left(T_{*}\right)$ if $T$ can be obtained from $T_{*}$ by a change of parameters, in precise terms, if there exists a homeomorphism $H(D)=D_{*}$ such that $T(p)=T_{*} H(p)$ for every point $p$ in $D$.

More generally, $A(T)=A\left(T_{*}\right)$ if $T$ is $F$-equivalent (equivalent in the Fréchet sense) to $T_{*}$, that is, if for every positive number $\epsilon$ there exists a homeomorphism $H_{\epsilon}(D)=D_{*}$ such that the distance in Euclidean 3-space between the points $T(p)$ and $T_{*} H_{\epsilon}(p)$ is less than $\epsilon$ for every point $p$ in $D$. Since two $F$-equivalent representations yield the same Fréchet surface, this is merely a restatement of the fact that the Lebesgue area is independent of the choice of representation for the surface (see 0.1). In the sequel we shall write $T \sim T_{*}(F)$ to indicate that $T$ and $T_{*}$ are $F$-equivalent. We shall also speak of the $F$-invariance of $A(T)$, meaning that $T \sim T_{*}(F)$ implies $A(T)$ $=A\left(T_{*}\right)$.

0.3. The principal result of this paper is to establish the relation $A(T)$ $=A\left(T_{*}\right)$ in a still more general situation; namely, when $T$ is $K$-equivalent to $T_{*}$ (in symbols, $T \sim T_{*}(K)$ ), that is, if $T$ and $T_{*}$ possess simultaneous monotone-light factorizations $T=L M$ and $T_{*}=L M_{*}$, where $M$ and $M_{*}$ are continu-

Presented to the Society, April 26, 1946; received by the editors June 10, 1946.

(1) Numbers in brackets refer to the bibliography at the end of the paper. 
ous monotone mappings from $D$ and $D_{*}$ (see 0.1 ) onto the same middle-space $\mathcal{X}$ and $L$ is a continuous light mapping from $\mathcal{X}$ into 3 -space [5, pp. 420-421].

J. W. T. Youngs $[9$, p. 784] has already established the $K$-invariance of the Lebesgue area in the 2-sphere case (that is, $T \sim T_{*}(K)$ implies $A(T)$ $=A\left(T_{*}\right)$ if $T$ and $T_{*}$ are defined on topological 2-spheres). However, in the 2-cell case a procedure analogous to Youngs' leads to complications.

In a recent paper containing important topological results, T. Radó [5, p. 423] stated that $A(T)$ is $K$-invariant in the 2-cell case, but he gave no proof since it appeared that his topological results would have to be supplemented by extensive analytic results. At Radó's suggestion, the author began a study of the problem and found, ultimately, that a relatively simple observation (see 0.6 ) reduced the problem to one which could be handled by standard analytic devices.

0.4. By means of the topology developed by Rado [5], the problem of proving that $A(T)$ is $K$-invariant in the 2-cell case can be reduced to the following special case (see 1.3, 1.4):

Show that $A(T)=A\left(T_{*}\right)$ if $T \sim T_{*}(K)$ (see 0.3) and the middle-space $\mathcal{Y C}$ is a topological 2-sphere, the image of the perimeter of $D$ under $M$ being a single point $\pi$ of $\mathcal{X}$ which is distinct from the point image $\pi_{*}$ of the perimeter of $D_{*}$ under $M_{*}$.

0.5. More topology of the same type (see 1.4) shows that if the image of the center of $D$ under $M$ is assumed to be $\pi_{*}$ (and this is no restriction since some interior point of $D$ has $\pi_{*}$ as its image), then the continuous monotone map $M_{*}$ can even be selected in the following particular way: $M_{*}=M I_{*}$ on $D_{*}-(0,0)$ and $M_{*}(0,0)=\pi$, where $I_{*}$ is the inversion from $D_{*}$ to $D$ defined by the equations

$$
I_{*}:\left\{\begin{array}{l}
u=\left(1-\rho_{*}\right) u_{*} / \rho_{*}, \\
v=\left(1-\rho_{*}\right) v_{*} / \rho_{*},
\end{array} \quad \rho_{*}=\left(u_{*}^{2}+v_{*}^{2}\right)^{1 / 2}, 0<\rho_{*} \leqq 1 .\right.
$$

0.6. In attempting to prove $A(T)=A\left(T_{*}\right)$ for the situation described in 0.4 and 0.5 , it was found that the middle-space $\mathcal{X}$ and the monotone-light factorizations of $T$ and $T_{*}$ were not needed. In fact the following theorem was established:

Theorem. If the image of the perimeter of $D$ under $T$ (see 0.1 ) is a single point $P$ in 3-space and $T_{*}=T I_{*}$ on $D_{*}-(0,0), T_{*}(0,0)=P$, then $A(T)=A\left(T_{*}\right)$.

Thus the net effect of the topology is to reduce the problem of proving the $K$-invariance of $A(T)$ to that of proving the invariance of $A(T)$ under the particular change of parameters given by the inversion $I_{*}$.

0.7. Chapter 1 of the paper is devoted to the topology required to perform the reduction described in 0.4 and 0.5 . A proof of the theorem of 0.6 is given in Chapter 2. In Chapter 3 the $K$-invariance of the lower area $a(T)[4$, p. 104 ; 5, p. 423] is established for the 2 -cell case. 


\section{TOPOLOGICAL PRELIMINARIES}

1.1. Let $T(D)=\mathscr{P}$ be a continuous mapping from $D\left[u^{2}+v^{2} \leqq 1\right]$ onto a subset $\mathcal{P}$ of Euclidean 3-space (see 0.1). Let $T=L M, M(D)=\mathscr{H}, L(\mathscr{H} C)=\mathscr{P}$ be a monotone-light factorization of $T$ (see 0.3 ), and let us assume that the middle-space $\mathcal{H}$ is not a dendrite. Let $C^{1}, \cdots, C^{n}, \cdots$ be the proper cyclic elements of $\mathcal{X}$ and, for each $n$, let $\mu^{n}(\mathscr{X})=C^{n}$ be the unique monotone retraction from $\mathcal{X}$ onto $C^{n}\left[5\right.$, p. 421]. Put $T^{n}=L \mu^{n} M$. Then the mappings $T^{1}, \cdots, T^{n}, \cdots$ are said to constitute the cyclic decomposition of $T[5$, p. 421 ]. Let $A(T), A\left(T^{1}\right), \cdots, A\left(T^{n}\right), \cdots$ denote the Lebesgue areas of the Fréchet surfaces determined by the representations $T, T^{1}, \cdots, T^{n}, \cdots$. Then Radó [6, V.2] has established the fundamental formula $A(T)=A\left(T^{1}\right)$ $+\cdots+A\left(T^{n}\right)+\cdots$. In other words, the Lebesgue area is cyclicly additive in the 2-cell case. The cyclic additivity of the Lebesgue area in the 2 -sphere case was first established by Youngs [9, p. 783].

1.2. It should be mentioned that Radó [5, Part 4] recently proved a general cyclic additivity theorem which applies to both the Lebesgue area and the lower area (see 3.1), and covers the 2-cell case and 2-sphere case simultaneously.

1.3. Now let $T_{*}\left(D_{*}\right)=\Phi$ be a continuous mapping from $D_{*}\left[u_{*}^{2}+v_{*}^{2} \leqq 1\right]$ onto $P$ which is $K$-equivalent to $T$ (see 0.3 ). Then there exist monotone-light factorizations $T=L M, T_{*}=L M_{*}, M(D)=\mathscr{H} \mathcal{C}, M_{*}\left(D_{*}\right)=\mathscr{H}(L(\mathcal{H})=\mathscr{P}$. Again assume $\mathcal{X}$ is not a dendrite and let the cyclic decompositions of $T$ and $T_{*}$ be $T^{1}, \cdots, T^{n}, \cdots$ and $T_{*}^{1}, \cdots, T_{*}^{n}, \cdots$, where $T^{n}=L \mu^{n} M, T_{*}^{n}=L \mu^{n} M_{*}^{*}$, and $\mu^{n}(\mathscr{X})=C^{n}$ (see 1.1). We note that $T^{n} \sim T_{*}^{n}(K)$ for every $n$. It follows from the cyclic additivity theorem (see 1.1) that $A(T)=A\left(T_{*}\right)$ if $A\left(T^{n}\right)=A\left(T_{*}^{n}\right)$ for every $n$. (If $\mathcal{X}$ is a dendrite, the cyclic decompositions of $T$ and $T_{*}$ are empty and $A(T)=0=A\left(T_{*}\right)[9$, p. 784].) Since every proper cyclic element of $\mathcal{X}$ is either a topological 2-cell or a topological 2-sphere [5, p. 430], two cases arise.

Case 1. $C^{n}$ is a topological 2-cell. In this case $T^{n} \sim T_{*}^{n}(K)$ implies $T^{n} \sim T_{*}^{n}(F)\left[5\right.$, p. 439] and, hence, $A\left(T^{n}\right)=A\left(T_{*}^{n}\right)$ (see 0.2).

Case 2. $C^{n}$ is a topological 2-sphere. In this case the perimeter of $D$ is mapped into a single point $\pi$ of $C^{n}$ by the monotone transformation $\mu^{n} M$ $\left[5\right.$, p. 430]. Likewise the image under $\mu^{n} M_{*}$ of the perimeter of $D_{*}$ is a single point $\pi_{*}$ of $C^{n}$. Two possibilities exist.

Case 2a. The points $\pi$ and $\pi_{*}$ coincide. In this situation $T^{n} \sim T_{*}^{n}(K)$ again implies $T^{n} \sim T_{*}^{n}(F)\left[5\right.$, p. 440] and, hence, $A\left(T^{n}\right)=A\left(T_{*}^{n}\right)$.

Case $2 \mathrm{~b}$. The points $\pi$ and $\pi_{*}$ are distinct. For this case $T^{n} \sim T_{*}^{n}(K)$ does not imply $T^{n} \sim T_{*}^{n}(F)$ in general; however, we still can prove that $A\left(T^{n}\right)$ $=A\left(T_{*}^{n}\right)$.

1.4. To simplify notations let us assume that the common middle-space $\mathcal{H}$ arising in the simultaneous monotone-light factorizations of $T$ and $T_{*}$ (see $0.3,1.3$ ) is itself a topological 2 -sphere. Let us also assume, corresponding to 
Case $2 \mathrm{~b}$ of 1.3 , that the image of the perimeter of $D$ under $M$ (see $0.3,1.3$ ) is a point $\pi$ of $\mathcal{X}$ which is distinct from the point image $\pi_{*}$ of the perimeter of $D_{*}$ under $M_{*}$. In this situation it is not a restriction to assume that the image of the center of $D$ under $M$ is the point $\pi_{*}$ since some interior point of $D$ has $\pi_{*}$ as its image under $M$.

As a first step toward establishing the relation $A(T)=A\left(T_{*}\right)$ for this case, let us consider a second continuous monotone map $\bar{M}_{*}$ from $D_{*}$ to $\mathcal{X}$ which is defined as follows: $\bar{M}_{*}=M I_{*}$ on $D_{*}-(0,0)$ and $\bar{M}_{*}(0,0)=\pi$, where $I_{*}$ is the inversion of 0.5 . Observe that $\pi_{*}$ is the image of the perimeter of $D_{*}$ under $\bar{M}_{*}$ as well as under $M_{*}$. Hence the transformation $\bar{T}_{*}=L \bar{M}_{*}$ is not only $K$-equivalent to $T_{*}$, but, by Case 2a of $1.3, \bar{T}_{*} \sim T_{*}(F)$ and $A\left(\bar{T}_{*}\right)=A\left(T_{*}\right)$. Thus in proving $A(T)=A\left(T_{*}\right)$ we may assume that $M_{*}$ itself is obtained from $M$ through the inversion $I_{*}$ (see 0.5 ). In Chapter 2 we shall prove the theorem of 0.6 , which covers this situation as a special case.

\section{Proof of the theorem of $\mathbf{0 . 6}$}

2.1. Let a continuous transformation $T$ from $D\left[u^{2}+v^{2} \leqq 1\right]$ onto a subset of Euclidean 3-space be given by the vector equation $\mathfrak{x}=\mathfrak{x}(u, v)$, where the components of $\mathfrak{x}(u, v)$ are $(x(u, v), y(u, v), z(u, v))$ (see 0.1). Using the complex variable $w=u+i v$, we may write $T: \mathfrak{x}=\mathfrak{x}(w), w \in D[|w| \leqq 1]$. When convenient, the vector $\mathfrak{x}=(x, y, z)$ will be considered as the point $(x, y, z)$ in 3-space.

2.2. Suppose that the surface represented by the continuous transformation $T$ passes close to a fixed point $P$ in 3 -space and it is desired to alter the given surface to make it pass through $P$ without changing the area too greatly. It seems natural in this situation to throw two concentric spheres about the point $P$ which intersect the surface and then to shrink the inner sphere and that portion of the surface lying within it down to the point $P$, at the same time stretching that part of the surface lying between the two spheres toward $P$ and leaving fixed that part lying outside the second sphere. Morrey [2, p. 315] suggested that a linear function of the distance to the point $P$ could be used to determine the amount that a point between the spheres should be moved toward $P$; however, Radó and Reichelderfer [7, pp. 649-650] pointed out that if the stretching is linear the area of the stretched surface is not close enough to that of the given surface for the application intended. On the other hand, they set up a one-parameter family of functions of the distance to the point $P$, each of which is a satisfactory "stretching function" for the applications. Presently we shall define and use one of their functions - the one which has the simplest analytic expression but actually is the least favorable from the point of view of changing the area [7, p. 649].

Let there be given two numbers $0<r<R$ and a fixed point $P$ in 3-space with coordinates $\left(x_{1}, y_{1}, z_{1}\right)$. We shall represent the point $P$ by the vector $\mathfrak{a}=\left(x_{1}, y_{1}, z_{1}\right)$. Then for $0 \leqq \rho<+\infty$ we define a continuous function $\lambda(\rho)$ by 


$$
\lambda(\rho)=\left\{\begin{array}{rr}
0 & \text { for } 0 \leqq \rho \leqq r, \\
R(\rho-r) / \rho(R-r) & \text { for } r \leqq \rho \leqq R, \\
1 & \text { for } R \leqq \rho<+\infty .
\end{array}\right.
$$

Finally we define a continuous vector function $\mathfrak{r}^{*}(w)$ by the formula $[6$, V.1 $]$

$$
\mathfrak{r}^{*}(w)=\mathfrak{a}+\lambda(|\mathfrak{x}(w)-\mathfrak{a}|)[\mathfrak{x}(w)-\mathfrak{a}], \quad w \in D,
$$

where $|\mathfrak{x}(w)-\mathfrak{a}|$ denotes the length of the vector $\mathfrak{x}(w)-\mathfrak{a}$. We shall say that $\mathfrak{r}^{*}(w)$ is obtained from $\mathfrak{x}(w)$ by the stretching operation $\Omega=\Omega(r, R, \mathfrak{a})$, in symbols $\mathfrak{x}^{*}(w)=\Omega \mathfrak{r}(w)=\Omega(r, R, \mathfrak{a}) \mathfrak{x}(w)$.

2.3. Under the stretching operator $\Omega(r, R, \mathfrak{a})$, those points $\mathfrak{x}=\mathfrak{x}(w)$ for which $|\mathfrak{x}(w)-\mathfrak{a}| \geqq R$ are not moved at all; points for which $r<|\mathfrak{x}(w)-\mathfrak{a}|<R$ are moved toward the point $\mathfrak{a}$; and points for which $|\mathfrak{x}(w)-\mathfrak{a}| \leqq r$ are carried in to the point $\mathfrak{a}$. It is clear, then, that $\left|\mathfrak{x}^{*}(w)-\mathfrak{r}(w)\right| \leqq R$ for $w \in D$.

2.4. It will be useful to know that the function $\lambda(\rho)$ of 2.2 satisfies a Lipschitz condition on $0 \leqq \rho<+\infty$. This follows immediately from the fact that $\lambda(\rho)$ is continuous on $0 \leqq \rho<+\infty$ and its derivative $\lambda^{\prime}(\rho)$ exists and is bounded on each of the three intervals $0<\rho<r, r<\rho<R, R<\rho<+\infty$. It is easily shown that $0 \leqq \lambda^{\prime}(\rho)<R /(R-r) r$ for $0<\rho<+\infty, \rho \neq r, R$; hence, for any two points $\rho_{1}, \rho_{2}$ in $0 \leqq \rho<+\infty$, we have $\left|\lambda\left(\rho_{2}\right)-\lambda\left(\rho_{1}\right)\right| \leqq R\left|\rho_{2}-\rho_{1}\right| /(R-r) r$.

2.5. A vector function $\mathfrak{x}(w)$ is said to satisfy a Lipschitz condition on the $\operatorname{disc} D$ if there exists a positive finite number $L$ such that $\left|\mathfrak{r}\left(w_{2}\right)-\mathfrak{r}\left(w_{1}\right)\right|$ $\leqq L\left|w_{2}-w_{1}\right|$ for any pair of points $w_{1}, w_{2}$ in $D$.

Lemma. If $\mathfrak{x}(w)$ satisfies a Lipschitz condition on $D$, then $\mathfrak{r}^{*}(w)=\Omega \mathfrak{r}(w)$ (see 2.2) also does.

Proof. Let $M$ be the maximum of $|\mathfrak{x}(w)-\mathfrak{a}|$ on $D$. For any two points $w_{1}, w_{2}$ in $D$ we have (see 2.2 )

$$
\begin{aligned}
\left|\mathfrak{r}^{*}\left(w_{2}\right)-\mathfrak{r}^{*}\left(w_{1}\right)\right|= & \mid \lambda\left(\left|\mathfrak{x}\left(w_{2}\right)-\mathfrak{a}\right|\right)\left[\mathfrak{x}\left(w_{2}\right)-\mathfrak{a}\right] \\
& -\lambda\left(\left|\mathfrak{x}\left(w_{1}\right)-\mathfrak{a}\right|\right)\left[\mathfrak{x}\left(w_{1}\right)-\mathfrak{a}\right] \mid \\
\leqq & \left|\lambda\left(\left|\mathfrak{x}\left(w_{2}\right)-\mathfrak{a}\right|\right)-\lambda\left(\left|\mathfrak{x}\left(w_{1}\right)-\mathfrak{a}\right|\right)\right|\left|\mathfrak{x}\left(w_{2}\right)-\mathfrak{a}\right| \\
& +\lambda\left(\left|\mathfrak{x}\left(w_{1}\right)-\mathfrak{a}\right|\right)\left|\mathfrak{x}\left(w_{2}\right)-\mathfrak{x}\left(w_{1}\right)\right| .
\end{aligned}
$$

By 2.4,

$$
\begin{aligned}
\left|\lambda\left(\left|\mathfrak{x}\left(w_{2}\right)-\mathfrak{a}\right|\right)-\lambda\left(\left|\mathfrak{x}\left(w_{1}\right)-\mathfrak{a}\right|\right)\right| & \\
& \leqq \frac{R}{(R-r) r}|| \mathfrak{x}\left(w_{2}\right)-\mathfrak{a}|-| \mathfrak{x}\left(w_{1}\right)-\mathfrak{a}|| \\
& \leqq \frac{R}{(R-r) r}\left|\mathfrak{x}\left(w_{2}\right)-\mathfrak{r}\left(w_{1}\right)\right| .
\end{aligned}
$$

Since $\mathfrak{x}(w)$ satisfies a Lipschitz condition on $D$ by assumption, there exists a 
finite constant $L$, independent of $w_{1}, w_{2}$, such that $\left|\mathfrak{x}\left(w_{2}\right)-\mathfrak{r}\left(w_{1}\right)\right| \leqq L\left|w_{2}-w_{1}\right|$. Therefore

$$
\begin{aligned}
\left|\mathfrak{x}^{*}\left(w_{2}\right)-\mathfrak{x}^{*}\left(w_{1}\right)\right| & \leqq\left[\frac{M R}{(R-r) r}+1\right]\left|\mathfrak{x}\left(w_{2}\right)-\mathfrak{x}\left(w_{1}\right)\right| \\
& \leqq\left[\frac{M R}{(R-r) r}+1\right] L\left|w_{2}-w_{1}\right| .
\end{aligned}
$$

This completes the proof.

2.6. Given a continuous vector function $\mathfrak{r}(w)$ on $D$, let us introduce the complex variable $w_{*}=u_{*}+i v_{*}$ and define a vector function $\mathfrak{x}_{*}\left(w_{*}\right)$ by the equation $\mathfrak{r}_{*}\left(w_{*}\right)=\mathfrak{r}\left(\tau\left(w_{*}\right)\right), 0<\left|w_{*}\right| \leqq 1$, where $w=\tau\left(w_{*}\right)=\left(1-\left|w_{*}\right|\right) w_{*} /\left|w_{*}\right|$, $0<\left|w_{*}\right| \leqq 1$, is the inversion $I_{*}$ of 0.5 . It is clear that we can define $\mathfrak{r}_{*}(0)$ in such a way as to make the extended function continuous on $D_{*}\left[\left|w_{*}\right| \leqq 1\right]$ if and only if the given function $\mathfrak{x}(w)$ is constant for $|w|=1$. If $\mathfrak{x}(w)$ is constant on the perimeter of $D$, we shall speak of the continuous vector function $\mathfrak{x}_{*}\left(w_{*}\right)=\mathfrak{r}\left(\tau\left(w_{*}\right)\right), w_{*} \in D_{*}$, even though $\tau\left(w_{*}\right)$ is not defined for $w_{*}=0$.

2.7. Lemma. If $\mathfrak{x}(w)$ is Lipschitzian on $D$ (see 2.5) and $\mathfrak{x}(w)=\mathfrak{a}$ on some annulus $1-\alpha \leqq|w| \leqq 1,0<\alpha \leqq 1$, then the continuous vector function $\mathfrak{x}_{*}\left(w_{*}\right)$ $=\mathfrak{x}\left(\tau\left(w_{*}\right)\right), w_{*} \in D_{*}($ see 2.6$)$, is Lipschitzian on $D_{*}$.

Proof. The inversion $w=\tau\left(w_{*}\right)$ is Lipschitzian on the annulus $0<\alpha \leqq\left|w_{*}\right|$ $\leqq 1$ and $\mathfrak{x}(w)$ is Lipschitzian on D by assumption; hence $\mathfrak{x}_{*}\left(w_{*}\right)$ is Lipschitzian on the annulus $0<\alpha \leqq\left|w_{*}\right| \leqq 1$. By assumption $\mathfrak{x}(w)=\mathfrak{a}$ for $1-\alpha \leqq|w| \leqq 1$, so $\mathfrak{r}_{*}\left(w_{*}\right)=\mathfrak{a}$ for $\left|w_{*}\right| \leqq \alpha$. It now follows that $\mathfrak{r}_{*}\left(w_{*}\right)$ is Lipschitzian on $D_{*}$.

2.8 . In the sequel we shall consider a vector function $\mathfrak{x}_{*}^{*}\left(w_{*}\right)$ which will be obtained from a given continuous vector function $\mathfrak{r}(w), w \in D$, by first applying the stretching operation $\Omega$ (see 2.2) to $\mathfrak{x}(w)$ and then changing parameters by means of the inversion $w=\tau\left(w_{*}\right)$ (see 2.6), in symbols $\mathfrak{r}_{*}^{*}\left(w_{*}\right)=\mathfrak{r}^{*}\left(\tau\left(w_{*}\right)\right)$, $0<\left|w_{*}\right| \leqq 1$, where $\mathfrak{r}^{*}(w)=\Omega \mathfrak{r}(w)$. Using the convention adopted in 2.6 , we shall speak of the continuous vector function $\mathfrak{r}_{*}^{*}\left(w_{*}\right)=\mathfrak{r}^{*}\left(\tau\left(w_{*}\right)\right), w_{*} \in D_{*}$, if $\mathfrak{r}^{*}(w)$ is constant for $|w|=1$.

2.9. Lemma. If $\mathfrak{x}(w)$ is Lipschitzian on $D$ (see 2.5) and $r>\max |\mathfrak{x}(w)-\mathfrak{a}|$ for $|w|=1$, then the continuous vector function, $\mathfrak{x}_{*}^{*}\left(w_{*}\right)=\mathfrak{r}^{*}\left(\tau\left(w_{*}\right)\right)$ (see 2.8), where $\mathfrak{x}^{*}(w)=\Omega(r, R, \mathfrak{a}) \mathfrak{x}(w)$ (see 2.2), is Lipschitzian on $D_{*}$.

Proof. By 2.5, $\mathfrak{x}^{*}(w)$ is Lipschitzian on $D$. Since $r>\max |\mathfrak{x}(w)-\mathfrak{a}|$ for $|w|=1, \mathfrak{x}^{*}(w)=\mathfrak{a}$ on some annulus $1-\alpha \leqq|w| \leqq 1,0<\alpha \leqq 1$ (see 2.3). Thus $\mathfrak{x}^{*}(w)$ satisfies the conditions of 2.7 and, hence, $\mathfrak{x}_{*}^{*}\left(w_{*}\right)$ is Lipschitzian on $D_{*}$.

2.10. Let $\mathfrak{x}(w)$ be a continuous vector function on $D$. If the first partial derivatives with respect to $u$ and $v$ of the components $x(u, v), y(u, v), z(u, v)$ of $\mathfrak{x}(w)$ (see 2.1) exist at some point $w=u+i v$ of $D^{0}$ (the set of interior points of $D$ ), we shall write: 


$$
\mathfrak{x}_{u}=\left(x_{u}, y_{u}, z_{u}\right), \quad \mathfrak{x}_{v}=\left(x_{v}, y_{v}, z_{v}\right), \quad W=\left[\mathfrak{x}_{u}^{2} \mathfrak{x}_{v}^{2}-\left(\mathfrak{x}_{u} \cdot \mathfrak{x}_{v}\right)^{2}\right]^{1 / 2} .
$$

The notations $\mathfrak{x}_{u}^{*}, \mathfrak{x}_{v}^{*}, W^{*}$ will have similar meanings with respect to the vector function $\mathfrak{r}^{*}(w)=\Omega \mathfrak{r}(w)$ (see 2.2).

2.11. Lemma. If $\mathfrak{x}(w)$ is continuous on $D, W$ exists at $w_{0} \in D^{0}$, and $\mathfrak{r}^{*}(w)$ $=\Omega(r, R, \mathfrak{a}) \mathfrak{r}(w)$, then $W^{*}$ exists at $w_{0}$ (providing $\left.\left|\mathfrak{x}\left(w_{0}\right)-\mathfrak{a}\right| \neq r, R\right)$ and $W^{*}\left(w_{0}\right) \leqq R W\left(w_{0}\right) /(R-r)$.

Proof. If either $\left|\mathfrak{x}\left(w_{0}\right)-\mathfrak{a}\right|<r$ or $\left|\mathfrak{x}\left(w_{0}\right)-\mathfrak{a}\right|>R$, the result is immediate from the definition of $\mathfrak{r}^{*}(w)$ (see 2.2, 2.3). If $r<\left|\mathfrak{x}\left(w_{0}\right)-\mathfrak{a}\right|<R$, the partial derivatives of $\mathfrak{x}^{*}(w)$ at $w_{0}$ can be computed by the chain rule. Then a direct computation shows that $[7$, p. 647$]$

$$
W^{* 2}=\lambda^{2}\left[\lambda^{2}+\lambda^{\prime} \rho\left(2 \lambda+\lambda^{\prime} \rho\right)\left\{W^{2}-\left(\frac{(\mathfrak{x}-\mathfrak{a}) \cdot\left(\mathfrak{x}_{u} \times \mathfrak{x}_{v}\right)}{\rho}\right)^{2}\right\}\right]
$$

where $\rho=\left|\mathfrak{x}\left(w_{0}\right)-\mathfrak{a}\right|$. Since $\lambda$ and $\lambda^{\prime}$ are positive for $r<\rho<R$ (see 2.2, 2.4), we obtain the desired inequality

$$
W^{*} / W \leqq \lambda\left(\lambda+\lambda^{\prime} \rho\right)=\left(\frac{R}{R-r}\right)^{2} \frac{\rho-r}{\rho} \leqq \frac{R}{R-r} .
$$

2.12. Lemma. If $\mathfrak{x}(w)$ is continuous on $D, W$ exists a.e. and is summable on $D$, and $\mathfrak{x}^{*}(w)=\Omega(r, R, \mathfrak{a}) \mathfrak{x}(w)$, then $W^{*}($ see 2.10$)$ exists a.e. and is summable on $D$ and

$$
\iint_{D} W^{*} \leqq \frac{R}{R-r} \iint_{D} W
$$

if $r$ and $R$ do not belong to a denumerable set $E=E(\mathfrak{x}, \mathfrak{a})$ of positive numbers.

Proof. For each positive number $a$, let $G_{a}$ be the set of those points of $D$ where $|\mathfrak{r}(w)-\mathfrak{a}|=a$. At most a denumerable number of the sets $G_{a}$ have positive measure, say $G_{a_{1}}, G_{a_{2}}, \cdots$. Let $E$ be the set $\left(a_{1}, a_{2}, \cdots\right)$. If $r$, $R \notin E$, then, since $W$ exists a.e. in $D$ by assumption, it follows from 2.11 that $W^{*}$ exists and satisfies the inequality $W^{*} \leqq R W /(R-r)$ a.e. in $D$. The assumed summability of $W$ on $D$ then implies the inequality

$$
\iint_{D} W^{*} \leqq \frac{R}{R-r} \iint_{D} W
$$

2.13. If the symbols $A(\mathfrak{x})$ and $A\left(\mathfrak{x}^{*}\right)$ are used to denote the Lebesgue areas of the Fréchet surfaces determined by the representations $\mathfrak{x}=\mathfrak{x}(w), w \in D$, and $\mathfrak{x}=\mathfrak{r}^{*}(w)=\Omega \mathfrak{r}(w), w \in D$ (see 2.2 ), we may state a useful corollary to the preceding lemma.

Corollary. If $\mathfrak{x}(w)$ is Lipschitzian on $D$, then $A\left(\mathfrak{x}^{*}\right) \leqq R A(\mathfrak{x}) /(R-r)$, providing $r, R \notin E=E(\mathfrak{x}, \mathfrak{a})$. 
Proof. Since $\mathfrak{x}(w)$ is Lipschitzian on $D, W$ exists a.e. and is summable on $D$, and $A(\mathfrak{x})=\iint_{D} W\left[3\right.$, p. 474]. By $2.5, \mathfrak{x}^{*}(w)$ is also Lipschitzian on $D$, so $A\left(\mathfrak{x}^{*}\right)=\iint_{D} W^{*}$. Hence, by 2.12 ,

$$
A\left(\mathfrak{x}^{*}\right)=\iint_{D} W^{*} \leqq \frac{R}{R-r} \iint_{D} W=\frac{R}{R-r} A(\mathfrak{x}),
$$

providing $r, R \notin E(\mathfrak{x}, \mathfrak{a})$.

REMARK. As a matter of fact, if $\mathfrak{x}(w)$ is continuous on $D$ then, for all positive numbers $0<r<R, A\left(\mathfrak{x}^{*}\right) \leqq R A(\mathfrak{x}) /(R-r)$. This general result, which we do not need, can be established readily by using quasi-linear approximations to the given vector function $\mathfrak{x}(w)$.

2.14. Lemma. If $\mathfrak{x}(w)$ is Lipschitzian on $D$ and $r>\max |\mathfrak{x}(w)-\mathfrak{a}|$ for $|w|=1$, then $A\left(\mathfrak{x}^{*}\right)=A\left(\mathfrak{x}_{*}^{*}\right)$, where $\mathfrak{x}^{*}(w)=\Omega(r, R, \mathfrak{a}) \mathfrak{x}(w), w \in D$, and $\mathfrak{x}_{*}^{*}\left(\mathfrak{w}_{*}\right)$ $=\mathfrak{r}^{*}\left(\tau\left(w_{*}\right)\right), w_{*} \in D_{*}($ see 2.8$)$.

Proof. By $2.5, \mathfrak{x}^{*}(w)$ is Lipschitzian on $D$ and, hence, $A\left(\mathfrak{x}^{*}\right)=\iint_{D} W^{*}$ (see 2.10). By $2.9, \mathfrak{x}_{*}^{*}\left(w_{*}\right)$ is Lipschitzian on $D_{*}$, so $A\left(\mathfrak{x}_{*}^{*}\right)=\iint_{D_{*}} W_{*}^{*}$, where (see 2.10) $W_{*}^{*}=\left[\mathfrak{r}_{* u}^{* 2} \mathfrak{r}_{* 0}^{* 2}-\left(\mathfrak{x}_{* u}^{*} \cdot \mathfrak{r}_{* 0}^{*}\right)^{2}\right]^{1 / 2}$.

Consider the annulus $A_{*}: 0<\alpha \leqq\left|w_{*}\right| \leqq \beta<1$ and its image $A: 1-\alpha \geqq|w|$ $\geqq 1-\beta$ under the inversion $w=\tau\left(w_{*}\right)$ (see 2.6). The inversion $w=\tau\left(w_{*}\right)$ is topological on $A_{*}$ and for a.e. $w_{*}$ in $A_{*}$ we have $W_{*}^{*}\left(w_{*}\right)=\left|J\left(w_{*}\right)\right| W^{*}\left(\tau\left(w_{*}\right)\right)$, where $J\left(w_{*}\right)$ is the Jacobian of the transformation $w=\tau\left(w_{*}\right)$. Therefore, by the ordinary formula for the transformation of double integrals [1, pp. 8384 ], we have

$$
\iint_{A} W^{*}(w)=\iint_{A_{*}} W^{*}\left(\tau\left(w_{*}\right)\right)\left|J\left(w_{*}\right)\right|=\iint_{A_{*}} W_{*}^{*}\left(w_{*}\right) .
$$

For $\alpha \rightarrow 0, \beta \rightarrow 1$, we obtain the result

$$
A\left(\mathfrak{x}^{*}\right)=\iint_{D} W^{*}=\iint_{D_{*}} W_{*}^{*}=A\left(\mathfrak{x}_{*}^{*}\right) .
$$

2.15. LemMA. If $\mathfrak{x}(w)$ is continuous on $D$, then there exists a sequence of Lipschitzian vector functions $\mathfrak{x}_{n}(w)$ on $D$ such that $\mathfrak{x}_{n}(w) \rightarrow \mathfrak{r}(w)$ uniformly on $D$ and $A\left(\mathfrak{x}_{n}\right) \rightarrow A(\mathfrak{x})$.

Proof. A similar lemma has been established by Youngs [8, p. 782] for a continuous vector function $\mathfrak{x}(w)$ defined on a square rather than a disc. By using a Lipschitzian homeomorphism from the unit disc to the unit square, the reader can easily extend Youngs' lemma to the one stated above.

2.16. LeMMA. If $\mathfrak{x}(w)$ is continuous on $D$ and $\mathfrak{x}(w)=\mathfrak{a}$ for $|w|=1$, then there exists a sequence of Lipschitzian vector functions $\mathfrak{y}_{n}\left(w_{*}\right), w_{*} \in D_{*}$, such that $\mathfrak{y}_{n}\left(w_{*}\right) \rightarrow \mathfrak{x}_{*}\left(w_{*}\right)=\mathfrak{x}\left(\tau\left(w_{*}\right)\right)$ (see 2.6) uniformly on $D_{*}$ and $\lim \sup A\left(\mathfrak{y}_{n}\right) \leqq A(\mathfrak{x})$. 
Proof. By 2.15 there exists a sequence of Lipschitzian vector functions $\mathfrak{x}_{n}(w)$ such that $\left|\mathfrak{x}(w)-\mathfrak{x}_{n}(w)\right|<1 / n^{2}$ for $w \in D$ and $A\left(\mathfrak{x}_{n}\right) \rightarrow A(\mathfrak{x})$. Let $E_{n}$ be the denumerable set $E\left(\mathfrak{x}_{n}, \mathfrak{a}\right)$ of 2.12 . Choose $r_{n}, R_{n} \notin E_{n}$ such that $\left|\mathfrak{r}(w)-\mathfrak{x}_{n}(w)\right|$ $<r_{n}<1 / n^{2}$ for $w \in D$ and $1 /(n+1)<R_{n}<1 / n$. Let $\mathfrak{x}_{n}{ }^{*}(w)=\Omega_{n} \mathfrak{x}_{n}(w)$, where $\Omega_{n}=\Omega\left(r_{n}, R_{n}, \mathfrak{a}\right)$. We assert that the sequence of Lipschitzian vector functions (see $2.8,2.9) \mathfrak{y}_{n}\left(w_{*}\right)=\mathfrak{r}_{n}^{*}\left(w_{*}\right)=\mathfrak{r}_{n}^{*}\left(\tau\left(w_{*}\right)\right), w_{*} \in D_{*}$, satisfies the requirements of the lemma.

Since $\mathfrak{x}(w)=\mathfrak{a}$ for $|w|=1$ and $\left|\mathfrak{x}(w)-\mathfrak{x}_{n}(w)\right|<r_{n}$ for $w \in D$, it follows that $\mathfrak{x}_{n}{ }^{*}(w)=\mathfrak{a}$ for $|w|=1$ (see 2.3). Hence $\mathfrak{y}_{n}(0)=\mathfrak{a}=\mathfrak{x}_{*}(0)$ for every $n$ (see 2.6, 2.8). For $w_{*} \neq 0$, it follows from 2.3 and the inequality $\left|\mathfrak{x}(w)-\mathfrak{x}_{n}(w)\right|<1 / n^{2}$, $w \in D$, that

$$
\begin{aligned}
\left|\mathfrak{y}_{n}\left(w_{*}\right)-\mathfrak{x}_{*}\left(w_{*}\right)\right| & =\left|\mathfrak{x}_{n}^{*}\left(\tau\left(w_{*}\right)\right)-\mathfrak{x}\left(\tau\left(w_{*}\right)\right)\right| \\
& \leqq\left|\mathfrak{x}_{n}^{*}\left(\tau\left(w_{*}\right)\right)-\mathfrak{x}_{n}\left(\tau\left(w_{*}\right)\right)\right|+\left|\mathfrak{x}_{n}\left(\tau\left(w_{*}\right)\right)-\mathfrak{x}\left(\tau\left(w_{*}\right)\right)\right| \\
& <R_{n}+1 / n^{2}<1 / n+1 / n^{2} .
\end{aligned}
$$

Therefore $\mathfrak{y}_{n}\left(w_{*}\right) \rightarrow \mathfrak{r}_{*}\left(w_{*}\right)$ uniformly on $D_{*}$.

By $2.13, A\left(\mathfrak{x}_{n}^{*}\right) \leqq R_{n} A\left(\mathfrak{x}_{n}\right) /\left(R_{n}-r_{n}\right)$. Since $R_{n} /\left(R_{n}-r_{n}\right) \rightarrow 1$ for $n \rightarrow \infty$, $\lim \sup A\left(\mathfrak{x}_{n}^{*}\right) \leqq \lim A\left(\mathfrak{x}_{n}\right)=A(\mathfrak{x})$. On the other hand, by $2.14, A\left(\mathfrak{y}_{n}\right)=A\left(\mathfrak{x}_{n}^{*}\right)$, so the proof is complete.

REMARK. The functions $\mathfrak{y}_{n}\left(w_{*}\right)$ were obtained by applying the stretching process to the functions $\mathfrak{x}_{n}(w)$ on $D$ and then using the inversion $w=\tau\left(w_{*}\right)$. It may be of interest to note that a sequence of vector functions satisfying the conditions of the above lemma can be acquired by first using the inversion to obtain a sequence of functions $\mathfrak{x}_{n *}\left(w_{*}\right)=\mathfrak{x}_{n}\left(\tau\left(w_{*}\right)\right.$ ) (all of which may be discontinuous at $w_{*}=0$ ) and then applying the stretching process to the functions $\mathfrak{x}_{n *}\left(w_{*}\right)$ on $D_{*}$. As a matter of fact, the lemma was first established in this way.

2.17. We are now ready to prove the theorem of 0.6. Using the vector notation of this chapter, the theorem may be stated as follows:

Theorem. If $\mathfrak{x}(w)$ is continuous on $D$ and $\mathfrak{x}(w)=\mathfrak{a}$ for $|w|=1$, then $A(\mathfrak{x})=A\left(\mathfrak{r}_{*}\right)$, where $\mathfrak{x}_{*}\left(w_{*}\right)$ is the continuous vector function $\mathfrak{x}\left(\tau\left(w_{*}\right)\right), w_{*} \in D_{*}$ (see 2.6).

Proof. Clearly the theorem will be established if we can show that the assumption $A(\mathfrak{x})<+\infty$ implies $A\left(\mathfrak{x}_{*}\right) \leqq A(\mathfrak{x})$ and the assumption $A\left(\mathfrak{x}_{*}\right)<+\infty$ implies $A(\mathfrak{x}) \leqq A\left(\mathfrak{x}_{*}\right)$.

Assume then that $A(\mathfrak{x})<+\infty$. By 2.16 there exists a sequence of Lipschitzian vector functions $\mathfrak{y}_{n}\left(w_{*}\right), w_{*} \in D_{*}$, converging uniformly to $\mathfrak{x}_{*}\left(w_{*}\right)$ on $D_{*}$ and such that $\lim \sup A\left(\mathfrak{y}_{n}\right) \leqq A(\mathfrak{x})$. Since $\mathfrak{y}_{n}\left(w_{*}\right) \rightarrow \mathfrak{r}_{*}\left(w_{*}\right)$ uniformly on $D_{*}$, it follows from the lower semi-continuity of the Lebesgue area that $A\left(\mathfrak{x}_{*}\right) \leqq \lim \inf A\left(\mathfrak{y}_{n}\right)$. Hence $A\left(\mathfrak{x}_{*}\right) \leqq \lim \inf A\left(\mathfrak{y}_{n}\right) \leqq \lim \sup A\left(\mathfrak{y}_{n}\right) \leqq A(\mathfrak{x})$. 
Now let us note the symmetry between $\mathfrak{x}(w)$ and $\mathfrak{r}_{*}\left(w_{*}\right)$. First of all, $\mathfrak{x}_{*}(0)=\mathfrak{a}$ and $\mathfrak{x}(w)=\mathfrak{a}$ for $|w|=1$. In addition, if $\mathfrak{x}(0)=\mathfrak{a}_{*}$, then $\mathfrak{x}_{*}\left(w_{*}\right)=\mathfrak{a}_{*}$ for $\left|w_{*}\right|=1$. We have regarded the continuous vector function $\mathfrak{x}_{*}\left(w_{*}\right)$ as being derived through the inversion $w=\tau\left(w_{*}\right)$ (see 2.6) from a given continuous vector function $\mathfrak{x}(w)$ such that $\mathfrak{x}(w)=\mathfrak{a}$ for $|w|=1$; however, we could ragard $\mathfrak{x}(w)$ as being derived through the inversion $w_{*}=\tau^{-1}(w)$ from a given continuous vector function $\mathfrak{r}_{*}\left(w_{*}\right)$ such that $\mathfrak{r}_{*}\left(w_{*}\right)=\mathfrak{a}_{*}$ for $\left|w_{*}\right|=1$.

It is apparent then from the symmetry just described that the inequality $A(\mathfrak{x}) \leqq A\left(\mathfrak{r}_{*}\right)$ can be obtained from the assumption $A\left(\mathfrak{r}_{*}\right)<+\infty$ by interchanging the roles of $\mathfrak{x}(w)$ and $\mathfrak{x}_{*}\left(w_{*}\right)$ in the proof given above for the complementary inequality.

\section{The $K$-Invariance of $a(T)$}

3.1. Let a Fréchet surface of the type of the 2 -cell be given by the representation (see 0.1) $T: x=x(u, v), y=y(u, v), z=z(u, v),(u, v) \in D$. With the continuous transformation $T$, we associate the three transformations (projections on the coordinate planes):

$$
\begin{aligned}
& T^{x}: \quad y=y(u, v), \quad z=z(u, v), \quad(u, v) \in D, \\
& T^{\nu}: \quad z=z(u, v), \quad x=x(u, v), \quad(u, v) \in D, \\
& T^{z}: \quad x=x(u, v), \quad y=y(u, v), \quad(u, v) \in D .
\end{aligned}
$$

The transformations $T^{x}, T^{y}, T^{z}$ give rise to the essential multiplicities [6, V.1] $\kappa\left(y, z ; T^{x}, \mathcal{D}\right), \kappa\left(z, x ; T^{y}, \mathcal{D}\right), \kappa\left(x, y ; T^{z}, \mathcal{D}\right)$, where $\mathcal{D}$ is any domain (connected open set) in $D^{0}$ (the set of interior points of $D$ ). We shall denote the integral of $\kappa\left(y, z ; T^{x}, \mathscr{D}\right)$ over the $y z$-plane by $g_{x}(D)$, if this integral exists in the Lebesgue sense; otherwise, we put $g_{x}(\mathscr{D})=+\infty$. The quantities $g_{y}(\mathcal{D})$ and $g_{z}(\mathscr{D})$ are defined similarly with respect to $\kappa\left(z, x ; T^{\nu}, \mathscr{D}\right)$ and $\kappa\left(x, y ; T^{z}, \mathscr{D}\right)$. Finally we put

$$
g(\mathcal{D})=\left(g_{x}(\mathcal{D})^{2}+g_{y}(\mathscr{D})^{2}+g_{z}(\mathcal{D})^{2}\right)^{1 / 2} .
$$

Let $\mathcal{D}_{1}, \mathcal{D}_{2}, \ldots$ be any finite or infinite system of disjoint domains in $D^{0}$. The lower area $a(T)$ is then defined to be the least upper bound of the summation $g\left(\mathcal{D}_{1}\right)+g\left(\mathcal{D}_{2}\right)+\cdots$ for all such systems $\mathcal{D}_{1}, \mathscr{D}_{2}, \cdots$.

3.2. The lower area $a(T)$ is independent of the choice of representation for the given Fréchet surface; in other words, $T \sim T_{*}(F)$ (see 0.2 ) implies $a(T)=a\left(T_{*}\right)[6, \mathrm{~V} .1]$. As mentioned in 1.2, $a(T)$ is also cyclicly additive $[6, \mathrm{~V} .1]$.

3.3. An examination of Chapter 1 shows that just two properties of the Lebesgue area $A(T)$ were used in carrying out the reduction of the problem of establishing the $K$-inveriance of $A(T)$ to the theorem of 0.6 . The two properties needed were $F$-invariance and cyclic additivity. Since the lower area $a(T)$ possesses these properties also (see 3.2), a similar reduction can be made 
for the problem of proving that $a(T)$ is $K$-invariant in the 2-cell case. We shall complete the proof by establishing the following theorem (see 0.6):

THEOREM. If the image of the perimeter of $D$ under $T$ (see 3.1) is a single point $P$ in 3-space and $T_{*}=T I_{*}$ on $D_{*}-(0,0)$ (see 0.5$), T_{*}(0,0)=P$, then $a(T)=a\left(T_{*}\right)$.

3.4. The theorem stated in 3.3 is a direct corollary of a useful lemma which we shall quote presently. First let $E$ be any closed set in $D^{0}$ and let $\mathcal{D}_{1}, \mathcal{D}_{2}, \cdots$ be any finite or infinite system of disjoint domains in $D^{0}-E$. Then let $G(T, D, E)$ be the least upper bound of the summation $g\left(\mathcal{D}_{1}\right)+g\left(\mathcal{D}_{2}\right)$ $+\ldots$ for all such systems $\mathcal{D}_{1}, \mathcal{D}_{2}, \ldots$ Radó $[6, V .1]$ has proved:

LEMMA. If each of the projections on the three coordinate planes of the image of $E$ under $T$ is a set of measure zero, then $G(T, D, E)=a(T)$

3.5. To show that the lemma of 3.4 implies the theorem of 3.3 , we first observe that if $E$ is the single point $(0,0)$ of $D^{0}$ and $E_{*}$ is the point $(0,0)$ of $D_{*}^{0}$ (the set of interior points of $D_{*}$ ), then $G(T, D, E)=a(T)$ and $G\left(T_{*}, D_{*}, E_{*}\right)$ $=a\left(T_{*}\right)$. Next let $\mathcal{D}$ be any domain in $D^{0}-E$ and let $\mathcal{D}_{*}=I_{*}^{-1}(\mathcal{D})$ (see 0.5 ). Then $\mathscr{D}_{*} \subset D_{*}^{0}-E_{*}$. Since $I_{*}$ is topological on $\mathcal{D}_{*}$, the essential multiplicities of 3.1 are not changed in value if the arguments $T^{x}, T^{y}, T^{z}$, and $\mathcal{D}$ are replaced by $T_{*}^{x}, T_{*}^{\nu}, T_{*}^{z}$, and $\mathcal{D}_{*}$ respectively [6, IV.1]. Hence $g\left(\mathcal{D}_{*}\right)=g(\mathcal{D})$ (see 3.1). Similarly, if $\mathscr{D}_{*}$ is any domain in $D_{*}^{0}-E_{*}$ and $\mathcal{D}=I_{*}\left(\mathcal{D}_{*}\right)$, then $\mathcal{D} \subset D^{0}-E$ and $g(\mathcal{D})=g\left(\mathcal{D}_{*}\right)$. Therefore $G(T, D, E)=G\left(T_{*}, D_{*}, E_{*}\right)$ (see 3.4) and the theorem of 3.3 follows from the equality $a(T)=G(T, D, E)=G\left(T_{*}, D_{*}, E_{*}\right)=a\left(T_{*}\right)$.

\section{BIBLIOGRAPHY}

1. R. G. Helsel and T. Rad6, The transformation of double integrals, Trans. Amer. Math. Soc. vol. 54 (1943) pp. 83-102.

2. C. B. Morrey, An analytic characterization of surfaces of finite Lebesgue area, Amer. J. Math vol. 58 (1936) pp. 312-322.

3. T. Rad6, Über das Flächenmass rekiifizierbarer Flächen, Math. Ann. vol. 100 (1928) pp. 445-479.

4. - On surface area, Proc. Nat. Acad. Sci. U.S.A. vol. 31 (1945) pp. 102-106.

5. - On continuous mappings of Peano spaces, Trans. Amer. Math. Soc. vol. 58 (1945) pp. $420-454$.

6. - Length and area, Amer. Math. Soc. Colloquium Publications, vol. 30, not yet published.

7. T. Rad6 and P. Reichelderfer, On a stretching process for surfaces, Amer. J. Math. vol. 61 (1939) pp. 645-650.

8. J. W. T. Youngs, The additivity of the Lebesgue area, Bull. Amer. Math. Soc. vol. 49 (1943) pp. 779-784. 785.

9. - The topological theory of Fréchet surfaces, Ann. of Math. vol. 45 (1944) pp. 753-

10. - Curves and surfaces, Amer. Math. Monthly vol. 51 (1944) pp. 1-11.

The Ohro State University, Columbus, Ohio. 